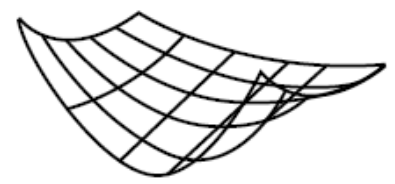

QUANTITATIVE FINANCE

RESEARCH CENTRE

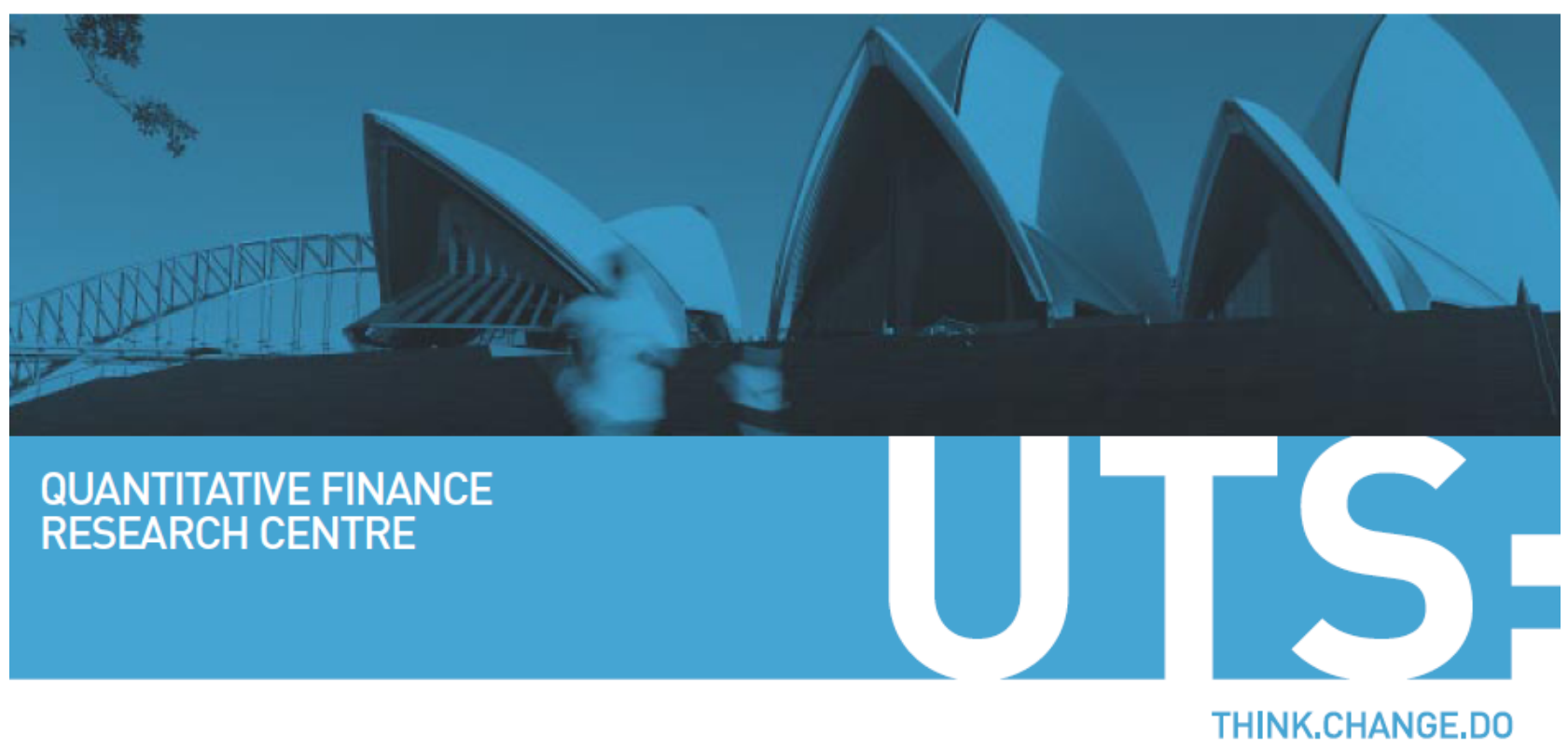

QUANTITATIVE FINANCE RESEARCH CENTRE

Affine Realizations for Lévy Driven Interest Rate Models with Real-World Forward Rate Dynamics Eckhard Platen and Stefan Tappe 


\title{
AFFINE REALIZATIONS FOR LÉVY DRIVEN INTEREST RATE MODELS WITH REAL-WORLD FORWARD RATE DYNAMICS
}

\author{
ECKHARD PLATEN AND STEFAN TAPPE
}

\begin{abstract}
We investigate the existence of affine realizations for interest rate term structure models driven by Lévy processes. Using as numéraire the growth optimal portfolio, we model the interest rate term structure under the realworld probability measure, and hence, we do not need the existence of an equivalent risk-neutral probability measure. Furthermore, we include finite dimensional external factors, thus admitting a stochastic volatility structure.
\end{abstract}

Key Words: Lévy driven interest rate models, real-world forward rate dynamics, stochastic volatility, affine realizations.

91B28, $60 \mathrm{H} 15$

\section{INTRODUCTION}

A zero coupon bond with maturity $T$ is a financial asset which pays the holder one unit of cash at $T$. Its price at $t \leq T$ can be written as the continuous discounting of one unit of cash

$$
P(t, T)=\exp \left(-\int_{t}^{T} f(t, s) d s\right),
$$

where $f(t, T)$ is the rate prevailing at time $t$ for instantaneous borrowing at time $T$, also called the forward rate for date $T$.

In the spirit of $[33,9]$, we model the forward rate dynamics as a jump-diffusion process under the real-world measure, which is driven by a process $X$ and based on the benchmark approach presented in [34]. More precisely, let $X$ be a real-valued, square-integrable Lévy process with Gaussian part $c \geq 0$ and Lévy measure $F$. Denoting by $\mu^{X}$ the random measure associated to the jumps of $X$, the dynamics of the unique generalized growth optimal portfolio $S^{*}$ are given by

$$
\begin{aligned}
d S_{t}^{*}= & S_{t}^{*}\left(r_{t}(0)+\theta_{t}^{2}+\int_{\mathbb{R}} \frac{\left(1-e^{x \theta_{t}}\right)^{2}}{e^{x \theta_{t}}} F(d x)\right) d t+\sqrt{c} S_{t}^{*} \theta_{t} d W_{t} \\
& +S_{t-}^{*} \int_{\mathbb{R}} \frac{1-e^{x \theta_{t-}}}{e^{x \theta_{t-}}}\left(\mu^{X}(d t, d x)-F(d x) d t\right),
\end{aligned}
$$

where $W$ is a Wiener process, $r_{t}(0)$ denotes the short rate, and $\theta$ denotes the market price of risk process. As in [33] or [9], using the growth optimal portfolio as numéraire, we price interest rate derivatives under the real-world probability measure, e.g.

$$
P(t, T)=\mathbb{E}\left[\frac{S_{t}^{*}}{S_{T}^{*}} \mid \mathcal{F}_{t}\right],
$$

and the real-world dynamics of the forward rates are of the form

$$
d f(t, T)=\alpha(t, T) d t+\sigma(t, T) d X_{t}, \quad t \in[0, T]
$$

Date: March 1, 2011. 
where the drift is given by

$$
\alpha(t, T)=\frac{d}{d T} \Psi\left(\theta_{t}-\int_{t}^{T} \sigma(t, s) d s\right)=-\sigma(t, T) \Psi^{\prime}\left(\theta_{t}-\int_{t}^{T} \sigma(t, s) d s\right),
$$

with $\Psi$ denoting the cumulant generating function of the Lévy process $X$. We refer the reader to [33] and [9] for more details on this topic.

Note that the real-world forward rate dynamics (1.1) are more general than Lévy driven term structure models under classical risk-neutral pricing, see Eberlein et al. $[18,17,13,14,15,16]$, which require the existence of an equivalent risk-neutral probability measure. In particular, we generalize the classical Heath, Jarrow and Morton (HJM) [24] equation and remove this assumption.

In the sequel, it will be convenient to switch under the real-world probability measure to the Musiela parametrization of forward curves $r_{t}(x)=f(t, t+x)$ (see [30]), and to consider the forward rates as the solution of a stochastic partial differential equation (SPDE), the so-called HJMM (Heath-Jarrow-Morton-Musiela) equation

$$
\left\{\begin{aligned}
d r_{t} & =\left(\frac{d}{d x} r_{t}+\alpha\left(r_{t}\right)\right) d t+\sigma\left(r_{t-}\right) d X_{t} \\
r_{0} & =h_{0},
\end{aligned}\right.
$$

on a suitable Hilbert space $H$ of forward curves, where $d / d x$ denotes the differential operator, which is generated by the strongly continuous semigroup $\left(S_{t}\right)_{t \geq 0}$ of shifts. Risk-neutral HJM equations of this kind have been investigated in $[20,32,29]$. Fundamental theoretical properties of equations of the type (1.3) are referred to in the just mentioned literature. Note that (1.3) does not represent a risk-neutral HJM equation and gives access to a wider modeling world than provided by the just mentioned papers.

We enlarge the term structure model (1.3) by adding an external, $\mathbb{R}^{m}$-valued factor process $Z$, admitting in this manner a stochastic volatility structure. Therefore, under the real-world probability measure we consider the HJMM equation

$$
\left\{\begin{aligned}
d r_{t} & =\left(\frac{d}{d x} r_{t}+\alpha\left(r_{t}, Z_{t}\right)\right) d t+\sigma\left(r_{t-}, Z_{t-}\right) d X_{t} \\
d Z_{t} & =b\left(r_{t}, Z_{t}\right) d t+c\left(r_{t-}, Z_{t-}\right) d X_{t} \\
r_{0} & =h_{0} \\
Z_{0} & =z_{0}
\end{aligned}\right.
$$

where, according to (1.2), the drift is given by

$$
\begin{aligned}
\alpha(h, z) & =\frac{d}{d x} \Psi\left(\theta(h, z)-\int_{0}^{\bullet} \sigma(h, z)(\eta) d \eta\right) \\
& =-\sigma(h, z) \Psi^{\prime}\left(\theta(h, z)-\int_{0}^{\bullet} \sigma(h, z)(\eta) d \eta\right), \quad(h, z) \in H \times \mathbb{R}^{m} .
\end{aligned}
$$

Risk-neutral Wiener driven HJM type equations with stochastic volatility have been studied in [7] and [22].

There are several reasons why, in practice, one is interested in the existence of a finite dimensional realization, that is, the forward rate evolution can be described by a finite dimensional state process. Such a finite dimensional realization ensures larger analytical tractability of the model, for example, in view of option pricing, see [12]. Moreover, as argued in [1], forward rate models without a finite dimensional realization do not seem reasonable, because then the support of the forward rate curves $f(t, t+\cdot), t>0$, becomes too large, and hence any "shape" of forward rate curves, which one assumes from the beginning to model the market phenomena, is destroyed with positive probability. 
For classical HJM equations driven by a Wiener process, the problem concerning the existence of a finite dimensional realization has been treated in $[27,35,12,2$, $26,4,5,10,11]$ and finally completely been solved in [8, 6, 21], see also [37], and [3] for a survey.

There are also some references, such as [18, 28, 23, 25, 38], that deal with affine realizations for term structure models with jumps.

The purpose of the present paper is to investigate the existence of affine realizations for real-world forward rate dynamics of the type (1.1), and to extend known results on this topic in the following directions:

- We study the geometric properties of forward rates as a whole under the benchmark approach.

- We do not need to assume the existence of an equivalent risk-neutral probability measure to obtain meaningful forward rate evolutions.

- Our models are driven by Lévy processes, which allows the occurrence of jumps with infinite intensity.

- We include finite dimensional external factors, thus, admitting a stochastic volatility structure.

In the present general framework we can, as in $[8,6,21,37,38]$, regard the problem under the real-world probability measure from a geometric point of view, i.e., the forward rate process has to stay on a finite dimensional submanifold $\mathcal{M}$. Since we deal with affine realizations, the submanifold is affine with the exception of one dimension that represents the time $t$. Hence, we may regard the submanifold $\mathcal{M}$ as a collection of affine manifolds indexed by the time $t$, which leads to the notion of an invariant foliation (see Section 2). From this view point, we shall derive all subsequent results in this text, which generalize those that have been derived under risk-neutral assumptions.

As we will see, with a driving Wiener process the term structure model has an affine realization if and only if the corresponding HJM equation under an assumed risk-neutral probability measure has an affine realization, see Proposition 4.1, and hence, all known results concerning the existence of affine realizations transfer to our present framework, which does not assume the existence of an equivalent riskneutral probability measure. In contrast, for a driving Lévy process with jumps we obtain further restrictions on the structure of the market price of risk, see Theorem 6.3 .

The remainder of this paper is organized as follows: In Section 2 we provide results on invariant foliations and on affine realizations for general SPDEs driven by Lévy processes. Afterwards, we introduce the term structure model in Section 3. After these preparations, we present general results on affine realizations for Wiener process driven term structure models in Section 4, and on Lévy term structure models in Section 5. Furthermore, we study constant direction volatilities in Section 6. Finally, Section 7 concludes.

\section{INVARIANT FOLIATIONS FOR STOCHASTIC PARTIAL DIFFERENTIAL EQUATIONS}

As indicated in the introduction, from a modeling point of view and also from the perspective of a compact and consistent characterization of forward rates, it is important to let these rates evolve in a description based on finite dimensional realizations of factor processes.

In this section, we provide results on invariant foliations for general SPDEs driven by Lévy processes, which we will apply to the HJMM equation (1.4) later on. In the sequel, we shall use the results from [37, Sec. 2,3] about invariant foliations. These results also hold true in our present framework with a driving Lévy process. If fact, due to the affine structure of a foliation, the Lévy process cannot jump out of the 
foliation. We also refer the reader to [37, Sec. 2,3] for more details and explanations about invariant foliations.

From now on, let $\left(\Omega, \mathcal{F},\left(\mathcal{F}_{t}\right)_{t \geq 0}, \mathbb{P}\right)$ be a filtered probability space satisfying the usual conditions and let $X$ be a real-valued, square-integrable Lévy process with Gaussian part $c \geq 0$ and Lévy measure $F$. In order to avoid trivialities, we assume that $c+F(\mathbb{R})>0$.

Here, we shall deal with SPDEs of the type

$$
\left\{\begin{aligned}
d r_{t} & =\left(\mathcal{A} r_{t}+\Theta\left(r_{t}\right)\right) d t+\Sigma\left(r_{t-}\right) d X_{t} \\
r_{0} & =h_{0}
\end{aligned}\right.
$$

on a separable Hilbert space $\mathcal{H}$. In (2.1), the operator $\mathcal{A}: \mathcal{D}(\mathcal{A}) \subset \mathcal{H} \rightarrow \mathcal{H}$ is the infinitesimal generator of a $C_{0}$-semigroup $\left(T_{t}\right)_{t>0}$ on $\mathcal{H}$ with adjoint operator $\mathcal{A}^{*}: \mathcal{D}\left(\mathcal{A}^{*}\right) \subset \mathcal{H} \rightarrow \mathcal{H}$. Recall that the domains $\mathcal{D}(\mathcal{A})$ and $\mathcal{D}\left(\mathcal{A}^{*}\right)$ are dense in $\mathcal{H}$, see, e.g., [36, Thm. 13.35.c, Thm. 13.12].

We call a $C_{0}$-semigroup $\left(T_{t}\right)_{t \geq 0}$ pseudo-contractive if there is a constant $\omega \geq 0$ such that

$$
\left\|T_{t}\right\| \leq e^{\omega t}, \quad t \geq 0 .
$$

Concerning the vector fields $\Theta, \Sigma: \mathcal{H} \rightarrow \mathcal{H}$ we impose the following conditions:

2.1. Assumption. We assume $\Theta, \Sigma \in C^{1}(\mathcal{H})$, that there exists a closed subspace $\mathcal{H}_{0} \subset \mathcal{H}$ such that $\Theta(\mathcal{H}) \subset \mathcal{H}_{0}, \Sigma(\mathcal{H}) \subset \mathcal{H}_{0}$, we have $T_{t} \mathcal{H}_{0} \subset \mathcal{H}_{0}$ for all $t \geq 0$ and $\left(T_{t}\right)_{t \geq 0}$ is pseudo-contractive on $\mathcal{H}_{0}$, and that there is a constant $L>0$ such that

$$
\begin{aligned}
\left\|\Theta\left(h_{1}\right)-\Theta\left(h_{2}\right)\right\| & \leq L\left\|h_{1}-h_{2}\right\|, \\
\left\|\Sigma\left(h_{1}\right)-\Sigma\left(h_{2}\right)\right\| & \leq L\left\|h_{1}-h_{2}\right\|
\end{aligned}
$$

for all $h_{1}, h_{2} \in \mathcal{H}$.

The Lipschitz assumptions (2.2), (2.3) ensure that for each $h_{0} \in \mathcal{H}$ there exists a unique weak solution for $(2.1)$ with $r_{0}=h_{0}$ having càdlàg sample paths, see $[20$, Thm. C.1].

In what follows, let $\mathcal{V} \subset \mathcal{H}$ be a finite dimensional linear subspace.

2.2. Definition. A family $\left(\mathcal{M}_{t}\right)_{t \geq 0}$ of affine subspaces $\mathcal{M}_{t} \subset \mathcal{H}, t \geq 0$ is called a foliation generated by $\mathcal{V}$ if there exists $\psi \in C^{1}\left(\mathbb{R}_{+} ; \mathcal{H}\right)$ such that

$$
\mathcal{M}_{t}=\psi(t)+\mathcal{V}, \quad t \geq 0 .
$$

The map $\psi$ is a parametrization of the foliation $\left(\mathcal{M}_{t}\right)_{t \geq 0}$.

2.3. Remark. Note that the parametrization of a foliation $\left(\mathcal{M}_{t}\right)_{t \geq 0}$ generated by $\mathcal{V}$ is not unique. However, due to condition (2.4), for two parametrizations $\psi_{1}, \psi_{2}$ we have

$$
\psi_{1}(t)-\psi_{2}(t) \in \mathcal{V} \quad \text { for all } t \geq 0 .
$$

In what follows, let $\left(\mathcal{M}_{t}\right)_{t \geq 0}$ be a foliation generated by $\mathcal{V}$.

2.4. Definition. For each $t \geq 0$ we define the tangent space

$$
T \mathcal{M}_{t}:=\psi^{\prime}(t)+\mathcal{V} \text {. }
$$

By Remark 2.3, the definition of the tangent is independent of the choice of the parametrization.

2.5. Definition. The foliation $\left(\mathcal{M}_{t}\right)_{t \geq 0}$ of submanifolds is invariant for (2.1) if for every $t_{0} \in \mathbb{R}_{+}$and $h \in \mathcal{M}_{t_{0}}$ we have

$$
\mathbb{P}\left(r_{t} \in \mathcal{M}_{t_{0}+t}\right)=1 \text { for all } t \geq 0,
$$

where $\left(r_{t}\right)_{t \geq 0}$ denotes the weak solution for (2.1) with $r_{0}=h$. 
We define the vector field $\mu: \mathcal{D}(\mathcal{A}) \rightarrow \mathcal{H}$ as

$$
\mu(h):=\mathcal{A} h+\Theta(h), \quad h \in \mathcal{D}(\mathcal{A}) .
$$

We have the following result concerning invariance of the foliation $\left(\mathcal{M}_{t}\right)_{t \geq 0}$ for the Lévy driven SPDE (2.1). Recall that $\mathcal{V} \subset \mathcal{H}$ denotes the finite dimensional linear subspace which generates the foliation $\left(\mathcal{M}_{t}\right)_{t \geq 0}$.

2.6. Theorem. The foliation $\left(\mathcal{M}_{t}\right)_{t \geq 0}$ is an invariant foliation for (2.1) if and only if for all $t \geq 0$ we have

$$
\begin{aligned}
\mathcal{M}_{t} & \subset \mathcal{D}(\mathcal{A}), \\
\mu(h) & \in T \mathcal{M}_{t}, \quad h \in \mathcal{M}_{t} \\
\Sigma(h) & \in \mathcal{V}, \quad h \in \mathcal{M}_{t} .
\end{aligned}
$$

Proof. See [37, Thm. 2.11].

Now, let $H$ be a separable Hilbert space, let $m \in \mathbb{N}$ be a positive integer, and set $\mathcal{H}=H \times \mathbb{R}^{m}$. Moreover, let $\alpha, \sigma: \mathcal{H} \rightarrow H$ and $b, c: \mathcal{H} \rightarrow \mathbb{R}^{m}$ be vector fields. Let $A: \mathcal{D}(A) \subset H \rightarrow H$ be the infinitesimal generator of a $C_{0}$-semigroup $\left(S_{t}\right)_{t \geq 0}$ on $H$ with adjoint operator $A^{*}: \mathcal{D}\left(A^{*}\right) \subset H \rightarrow H$. We consider the SPDE

$$
\left\{\begin{aligned}
d r_{t} & =\left(A r_{t}+\alpha\left(r_{t}, Z_{t}\right)\right) d t+\sigma\left(r_{t-}, Z_{t-}\right) d X_{t} \\
d Z_{t} & =b\left(r_{t}, Z_{t}\right) d t+c\left(r_{t-}, Z_{t-}\right) d X_{t} \\
r_{0} & =h_{0} \\
Z_{0} & =z_{0} .
\end{aligned}\right.
$$

Obviously, the SPDE (2.9) is of the type (2.1) with

$$
\mathcal{A}=\left(\begin{array}{cc}
A & 0 \\
0 & 0
\end{array}\right), \quad \Theta(h, z)=\left(\begin{array}{c}
\alpha(h, z) \\
b(h, z)
\end{array}\right), \quad \Sigma(h, z)=\left(\begin{array}{c}
\sigma(h, z) \\
c(h, z)
\end{array}\right) .
$$

We suppose that Assumption 2.1 is fulfilled. Let $V \subset H$ be a finite dimensional linear subspace, and let $\left(M_{t}\right)_{t \geq 0}$ be a foliation generated by $V$. We set $d:=\operatorname{dim} V$ and define $\nu: \mathcal{D}(A) \times \mathbb{R}^{m} \rightarrow H$ as

$$
\nu(h, z):=A h+\alpha(h, z) .
$$

2.7. Corollary. The foliation $\left(M_{t} \times \mathbb{R}^{m}\right)_{t \geq 0}$ is an invariant foliation for (2.9) if and only if for all $t \geq 0$ we have

$$
\begin{aligned}
M_{t} & \subset \mathcal{D}(A), \\
\nu(h, z) & \in T M_{t}, \quad(h, z) \in M_{t} \times \mathbb{R}^{m} \\
\sigma(h, z) & \in V, \quad(h, z) \in M_{t} \times \mathbb{R}^{m} .
\end{aligned}
$$

Proof. This is an immediate consequence of Theorem 2.6.

Now, we express the consistency conditions from Corollary 2.7 by means of a coordinate system. Let $\psi \in C^{1}\left(\mathbb{R}_{+} ; H\right)$ be a parametrization of $\left(M_{t}\right)_{t \geq 0}$ and let $\left\{\lambda_{1}, \ldots, \lambda_{d}\right\}$ be a basis of $V$.

2.8. Corollary. The following statements are equivalent:

(1) $\left(M_{t} \times \mathbb{R}^{m}\right)_{t \geq 0}$ is an invariant foliation for (2.9).

(2) We have

$$
\begin{aligned}
\psi\left(\mathbb{R}_{+}\right) & \subset \mathcal{D}(A), \\
\lambda_{1}, \ldots, \lambda_{d} & \in \mathcal{D}(A)
\end{aligned}
$$


and there exist $\mu, \gamma: \mathbb{R}_{+} \times \mathbb{R}^{d} \times \mathbb{R}^{m} \rightarrow \mathbb{R}^{d}$ such that

$$
\nu\left(\psi(t)+\sum_{i=1}^{d} y_{i} \lambda_{i}, z\right)=\psi^{\prime}(t)+\sum_{i=1}^{d} \mu_{i}(t, y, z) \lambda_{i}, \quad(t, y, z) \in \mathbb{R}_{+} \times \mathbb{R}^{d} \times \mathbb{R}^{m}
$$

$$
\sigma\left(\psi(t)+\sum_{i=1}^{d} y_{i} \lambda_{i}, z\right)=\sum_{i=1}^{d} \gamma_{i}(t, y, z) \lambda_{i}, \quad(t, y, z) \in \mathbb{R}_{+} \times \mathbb{R}^{d} \times \mathbb{R}^{m} .
$$

If the previous conditions are satisfied, then $\mu$ and $\gamma$ are uniquely determined, and we have $\mu, \gamma \in C^{0,1,1}\left(\mathbb{R}_{+} \times \mathbb{R}^{d} \times \mathbb{R}^{m} ; \mathbb{R}^{d}\right)$.

Proof. This is a direct consequence of Corollary 2.7.

The previous results lead to the following definition of an affine realization:

2.9. Definition. Let $V \subset H$ be a finite dimensional linear subspace. The SPDE (2.9) has an affine realization generated by $V$ if for each $\left(h_{0}, z_{0}\right) \in \mathcal{D}(A) \times \mathbb{R}^{m}$ there exists a foliation $\left(M_{t}^{\left(h_{0}, z_{0}\right)}\right)_{t \geq 0}$ generated by $V$ with $h_{0} \in M_{0}^{\left(h_{0}, z_{0}\right)}$, such that $\left(M_{t}^{\left(h_{0}, z_{0}\right)} \times \mathbb{R}^{m}\right)_{t \geq 0}$ is an invariant foliation for (2.9).

We call $d:=\operatorname{dim} V$ the dimension of the affine realization.

2.10. Lemma. Let $d \in \mathbb{N}$ and $\lambda_{1}, \ldots, \lambda_{d} \in H$ be linearly independent. Suppose the SPDE (2.1) has a d-dimensional affine realization generated by $V=\left\langle\lambda_{1}, \ldots, \lambda_{d}\right\rangle$. Then, there exist $\Phi_{1}, \ldots, \Phi_{d} \in C^{1}(\mathcal{H} ; \mathbb{R})$ such that

$$
\sigma(h, z)=\sum_{i=1}^{d} \Phi_{i}(h, z) \lambda_{i}, \quad(h, z) \in \mathcal{H}
$$

Proof. Relation (2.13) from Corollary 2.7 yields $\sigma(h, z) \in V$ for all $(h, z) \in \mathcal{D}(A) \times$ $\mathbb{R}^{m}$. Since $\mathcal{D}(A)$ is dense in $H$ and $V$ is closed, we obtain $\sigma(h, z) \in V$ for all $(h, z) \in \mathcal{H}$. Hence, there exist $\Phi_{1}, \ldots, \Phi_{d}: \mathcal{H} \rightarrow \mathbb{R}$ such that (2.18) is satisfied. Since $\sigma \in C^{1}(\mathcal{H} ; H)$, we have $\Phi_{1}, \ldots, \Phi_{d} \in C^{1}(\mathcal{H} ; \mathbb{R})$.

An element $h \in \mathcal{D}\left(A^{\infty}\right)$ is called quasi-exponential if

$$
\operatorname{dim}\left\langle A^{n} h: n \in \mathbb{N}_{0}\right\rangle<\infty,
$$

where $\mathbb{N}_{0}=\{0,1, \ldots\}$ denotes the set of all nonnegative integers. Quasi-exponential functions will appear later. We recall the following result:

2.11. Lemma. Let $h \in H$ be arbitrary. The following statements are equivalent:

(1) $h$ is quasi-exponential.

(2) There exists $d \in \mathbb{N}$ such that $h \in \mathcal{D}\left(A^{d}\right)$ and $A^{d} h \in\left\langle h, A h, \ldots, A^{d-1} h\right\rangle$.

(3) There exists a finite dimensional subspace $V \subset \mathcal{D}(A)$ with $h \in V$ such that

$$
A v \in V \quad \text { for all } v \in V .
$$

Proof. See [37, Lemma 3.6].

\section{Presentation of the term structure Model}

We shall now introduce the Lévy term structure model. Recall that $c \geq 0$ denotes the Gaussian part and $F$ the Lévy measure of the Lévy process $X$.

3.1. Assumption. We assume there exist constants $N, \epsilon>0$ such that

$$
\int_{\{|x|>1\}} e^{z x} F(d x)<\infty, \quad z \in[-(1+\epsilon) N,(1+\epsilon) N] .
$$


Then, the Lévy process $X$ possesses moments of arbitrary order and the cumulant generating function $\Psi(z):=\ln \mathbb{E}\left[e^{z X_{1}}\right]$ exists on $[-(1+\epsilon) N,(1+\epsilon) N]$ and has the representation

$$
\Psi(z)=b z+\frac{c}{2} z^{2}+\int_{\mathbb{R}}\left(e^{z x}-1-z x\right) F(d x), \quad z \in[-(1+\epsilon) N,(1+\epsilon) N],
$$

where $b \in \mathbb{R}$ denotes the drift of $X$. By (3.1) and Lebesgue's dominated convergence theorem, the cumulant generating function belongs to class $C^{\infty}$ on the open interval $(-(1+\epsilon) N,(1+\epsilon) N)$. In fact, $\Psi$ is even analytic on $(-(1+\epsilon) N,(1+\epsilon) N)$, and thus, we obtain

$$
\Psi^{\prime}(z)=\sum_{n=0}^{\infty} \frac{\Psi^{(n+1)}(0)}{n !} z^{n}, \quad z \in(-(1+\epsilon) N,(1+\epsilon) N) .
$$

For $\beta>0$ let $H_{\beta}$ be the space of all absolutely continuous functions $h: \mathbb{R}_{+} \rightarrow \mathbb{R}$ such that

$$
\|h\|_{\beta}=\left(|h(0)|^{2}+\int_{\mathbb{R}_{+}}\left|h^{\prime}(x)\right|^{2} e^{\beta x} d x\right)^{1 / 2}<\infty,
$$

and set $\mathcal{H}_{\beta}=H_{\beta} \times \mathbb{R}^{m}$. By [37, Thm. 4.1], the space $H_{\beta}$ is a separable Hilbert space and the subspace $H_{\beta}^{0}=\left\{h \in H_{\beta}: h(\infty)=0\right\}$ is closed. We fix constants $0<\beta<\beta^{\prime}$, a volatility $\sigma: \mathcal{H}_{\beta} \rightarrow H_{\beta}$, a market price of risk $\theta: \mathcal{H}_{\beta} \rightarrow \mathbb{R}$ and $b, c: \mathcal{H}_{\beta} \rightarrow \mathbb{R}^{m}$. Note that every constant function belongs to $H_{\beta}$, whence in the specification of $\theta$ we may regard $\mathbb{R}$ as a subspace of $H_{\beta}$. We define the open set $\mathcal{O} \subset H_{\beta}$ as

$$
\mathcal{O}:=\left\{h \in H_{\beta}:\|h\|_{\beta}<\frac{N}{C}\right\}
$$

where the constant $C>0$ stems from the estimate

$$
\|h\|_{L^{\infty}\left(\mathbb{R}_{+}\right)} \leq C\|h\|_{\beta}, \quad h \in H_{\beta},
$$

see [37, Thm. 4.1]. By this estimate, for all $h \in \mathcal{O}$ we have $\|h\|_{L^{\infty}\left(\mathbb{R}_{+}\right)} \leq N$. For $\lambda \in H_{\beta}$ we set $\Lambda:=\mathcal{I} \lambda:=\int_{0}^{\bullet} \lambda(\eta) d \eta$.

In the sequel, for two normed spaces $H$ and $G$ the space $C_{\text {lip }}^{1}(H ; G)$ denotes the space of all continuously differentiable Lipschitz functions, and $C_{b}^{1}(H ; G) \subset$ $C_{\text {lip }}^{1}(H ; G)$ denotes the space of all continuously differentiable bounded functions with bounded derivative.

3.2. Assumption. We have $\sigma \in C_{b}^{1}\left(\mathcal{H}_{\beta} ; H_{\beta}\right)$ and $\theta \in C_{b}^{1}\left(\mathcal{H}_{\beta} ; \mathbb{R}\right)$ with $\sigma\left(\mathcal{H}_{\beta}\right) \subset H_{\beta^{\prime}}^{0}$ and $(\theta-\mathcal{I} \sigma)\left(\mathcal{H}_{\beta}\right) \subset \mathcal{O}$. Furthermore, we assume that $b, c \in C_{\text {lip }}^{1}\left(\mathcal{H}_{\beta} ; \mathbb{R}^{m}\right)$.

We define the drift $\alpha: \mathcal{H}_{\beta} \rightarrow H_{\beta}$ according to (1.5). Note that the HJMM equation (1.4) is a particular example of the $\operatorname{SPDE}(2.9)$ with $A=d / d x$. Arguing as in [20, Prop. 4.5], the vector field $\alpha$ is Lipschitz continuous, and we have $\alpha\left(\mathcal{H}_{\beta}\right) \subset H_{\beta}^{0}$. Moreover, by [37, Lemmas 4.2, 4.3] we have $\alpha \in C^{1}\left(\mathcal{H}_{\beta} ; H_{\beta}\right)$. Hence, all required conditions from Assumption 2.1 are fulfilled, which ensures existence and uniqueness of weak solutions for the HJMM equation (1.4)

\section{Affine Realizations For Wiener term STRUCture models}

We are now ready to present some results regarding the existence of affine realizations for interest rate term structure models considered under the real-world probability measure. In this section, we study the particular situation where $X$ is a 
standard Wiener process $W$. Then, the cumulant generating function (3.2) is given by $\Psi(z)=\frac{1}{2} z^{2}$, and hence, the drift term (1.5) has the representation

$$
\alpha(h, z)=-\theta(h, z) \sigma(h, z)+\sigma(h, z) \int_{0}^{\bullet} \sigma(h, z)(\eta) d \eta .
$$

In particular, if $\theta \equiv 0$, then the drift is given by

$$
\alpha(h, z)=\sigma(h, z) \int_{0}^{\bullet} \sigma(h, z)(\eta) d \eta .
$$

Note that (4.2) is just the classical HJM drift condition of a Wiener process driven term structure model under a risk-neutral measure.

4.1. Proposition. The HJMM equation (1.4) has an affine realization if and only if the HJMM equation (1.4) with $\theta \equiv 0$ has an affine realization.

Proof. Let $\left(M_{t}\right)_{t \geq 0}$ be an arbitrary foliation generated by some finite dimensional subspace $V \subset H_{\beta}$ such that conditions (2.11), (2.13) are satisfied. Then, condition (2.12) with $\alpha$ given by (4.1) is fulfilled if and only if we have (2.12) with $\alpha$ given by (4.2). Therefore, Corollary 2.7 concludes the proof.

Consequently, we can draw the following important conclusion: All known results concerning the existence of affine realizations for HJM interest rate models under a risk-neutral probability measure (see [8, 6, 21, 7, 22, 37]) transfer to interest rate models with real-world forward rate dynamics.

Now, let the volatility $\sigma$ be of the form

$$
\sigma(h, z)=\sum_{i=1}^{p} \Phi_{i}(h, z) \lambda_{i}, \quad(h, z) \in \mathcal{H}_{\beta},
$$

where $p \in \mathbb{N}$ denotes a positive integer, $\Phi_{1}, \ldots, \Phi_{p}: \mathcal{H}_{\beta} \rightarrow \mathbb{R}$ are functionals and $\lambda_{1}, \ldots, \lambda_{d} \in H_{\beta^{\prime}}^{0}$ are linearly independent. We assume that $\Phi_{i} \in C_{b}^{1}\left(\mathcal{H}_{\beta} ; \mathbb{R}\right)$ for $i=1, \ldots, d$ and the inclusion $(\theta-\mathcal{I} \sigma)\left(\mathcal{H}_{\beta}\right) \subset \mathcal{O}$. Then, Assumption 3.2 is fulfilled.

Note that, in view of Lemma 2.10, this is the most general volatility, which we can have for the HJMM equation (1.4) with an affine realization.

4.2. Proposition. If $\lambda_{1}, \ldots, \lambda_{p}$ are quasi-exponential, then the HJMM equation (1.4) has an affine realization.

Proof. Due to Proposition 4.1 it suffices to consider the case $\theta \equiv 0$. By [37, Prop. 6.2] (and its proof) there exists a finite dimensional subspace $V \subset \mathcal{D}(d / d x)$ such that for all $\left(h_{0}, z_{0}\right) \in \mathcal{D}(d / d x) \times \mathbb{R}^{m}$ the foliation $M_{t}^{h_{0}}=S_{t} h_{0}+V, t \geq 0$ is an invariant foliation for the SPDE

$$
\left\{\begin{aligned}
d r_{t} & =\left(\frac{d}{d x} r_{t}+\alpha\left(r_{t}, z_{0}\right)\right) d t+\sigma\left(r_{t}, z_{0}\right) d W_{t} \\
r_{0} & =h_{0} .
\end{aligned}\right.
$$

Applying Theorem 2.6 yields that conditions (2.11)-(2.13) are satisfied. Thus, Corollary 2.7 implies that $\left(M_{t}^{h_{0}} \times \mathbb{R}^{m}\right)_{t \geq 0}$ is an invariant foliation for the HJMM equation (1.4), which finishes the proof.

Note that Proposition 4.2 is a practically rather important statement. As an example, let $\alpha_{0}, \eta>0$ be constants and consider the interest rate model

$$
\left\{\begin{aligned}
d r_{t} & =\left(\frac{d}{d x} r_{t}+\alpha\left(r_{t}, Z_{t}\right)\right) d t+\sigma\left(r_{t}, Z_{t}\right) d W_{t} \\
d Z_{t} & =\left(1-\eta Z_{t}\right) d t+\sqrt{Z_{t}} d W_{t} \\
r_{0} & =h_{0} \\
Z_{0} & =S_{0}^{*} / \alpha_{0},
\end{aligned}\right.
$$


with market price of risk $\theta(h, z)=1 / \sqrt{z}$. Then, the growth optimal portfolio is that of a minimal market model, see [34]. The stochastic factor process $Z$ is a time transformed square root process of dimension four, and we can express the growth optimal portfolio as

$$
S_{t}^{*}=\alpha_{0} e^{\eta t} Z_{t} \exp \left(\int_{0}^{t} r_{s}(0) d s\right), \quad t \geq 0 .
$$

By Proposition 4.2, for any volatility $\sigma$ of the form (4.3) with $\lambda_{1}, \ldots, \lambda_{p}$ being quasi-exponential the HJMM equation (4.4) has an affine realization.

\section{Affine ReAlizations For LÉvy term STRUCture MODElS}

We shall now provide some general results regarding affine realizations for Lévy term structure models. Our results from this section generalize those of [38, Sec. 3], where the risk-neutral situation corresponding to $\theta \equiv 0$ without stochastic volatility was considered.

We assume that the volatility $\sigma$ in the HJMM equation (1.4) is of the form

$$
\sigma(h, z)=\sum_{i=1}^{d} \Phi_{i}(h, z) \lambda_{i}, \quad(h, z) \in \mathcal{H}_{\beta},
$$

where $d \in \mathbb{N}$ denotes a positive integer, $\Phi_{1}, \ldots, \Phi_{d}: \mathcal{H}_{\beta} \rightarrow \mathbb{R}$ are functionals and $\lambda_{1}, \ldots, \lambda_{d} \in H_{\beta^{\prime}}^{0}$ are linearly independent. We assume that $\Phi_{i} \in C_{b}^{1}\left(\mathcal{H}_{\beta} ; \mathbb{R}\right)$ for $i=1, \ldots, d$ and the inclusion $(\theta-\mathcal{I} \sigma)\left(H_{\beta}\right) \subset \mathcal{O}$. Then, Assumption 3.2 is fulfilled.

Note that, in view of Lemma 2.10, this is the most general volatility, which we can have for the HJMM equation (1.4) with an affine realization. The corresponding drift term from (1.5) is given by

$$
\begin{aligned}
\alpha_{\mathrm{HJM}}(h, z) & =\frac{d}{d x} \Psi\left(\theta(h, z)-\sum_{i=1}^{d} \Phi_{i}(h, z) \Lambda_{i}\right) \\
& =-\sum_{i=1}^{d} \Phi_{i}(h, z) \lambda_{i} \Psi^{\prime}\left(\theta(h, z)-\sum_{i=1}^{d} \Phi_{i}(h, z) \Lambda_{i}\right), \quad(h, z) \in \mathcal{H}_{\beta} .
\end{aligned}
$$

5.1. Lemma. Suppose $\sigma \not \equiv 0$, that for all $i=1, \ldots, d$ and $(h, z) \in \mathcal{H}_{\beta}$ we have

$$
\begin{aligned}
& D \Phi_{i}(h, z)\left(\lambda_{j}, 0\right)=0, \quad j=1, \ldots, d \\
& D \Phi_{i}(h, z)\left(0, e_{k}\right)=0, \quad k=1, \ldots, m
\end{aligned}
$$

and for all $(h, z) \in \mathcal{H}_{\beta}$ with $\left(\Phi_{1}(h, z), \ldots, \Phi_{d}(h, z)\right) \neq 0$ we have

$$
\begin{aligned}
& D \theta(h, z)\left(\lambda_{j}, 0\right)=0, \quad j=1, \ldots, d \\
& D \theta(h, z)\left(0, e_{k}\right)=0, \quad k=1, \ldots, m .
\end{aligned}
$$

Then, the HJMM equation (1.4) has an affine realization generated by $\left\langle\lambda_{1}, \ldots, \lambda_{d}\right\rangle$ if and only if we have

$$
\begin{aligned}
& \lambda_{1}, \ldots, \lambda_{d} \in \mathcal{D}(d / d x), \\
& (d / d x) \lambda_{i} \in\left\langle\lambda_{1}, \ldots, \lambda_{d}\right\rangle, \quad i=1, \ldots, d .
\end{aligned}
$$

Proof. Suppose the HJMM equation (1.4) has an affine realization generated by $\left\langle\lambda_{1}, \ldots, \lambda_{d}\right\rangle$. Since $\sigma \not \equiv 0$ and $\mathcal{D}(d / d x)$ is dense in $H_{\beta}$, there exists $\left(h_{0}, z_{0}\right) \in$ $\mathcal{D}(d / d x) \times \mathbb{R}^{m}$ with $\sigma\left(h_{0}, z_{0}\right) \neq 0$. Applying Corollary 2.8 to the invariant foliation $\left(M_{t}^{\left(h_{0}, z_{0}\right)} \times \mathbb{R}^{m}\right)_{t \geq 0}$, we have $(5.7)$, and there exist $h_{0}^{\prime} \in H_{\beta}$ and $\mu, \gamma: \mathbb{R}^{d} \rightarrow \mathbb{R}^{d}$ 
such that

$$
\begin{aligned}
& \nu\left(h_{0}+\sum_{i=1}^{d} y_{i} \lambda_{i}, z_{0}\right)=h_{0}^{\prime}+\sum_{i=1}^{d} \mu_{i}(y) \lambda_{i}, \quad y \in \mathbb{R}^{d} \\
& \sigma\left(h_{0}+\sum_{i=1}^{d} y_{i} \lambda_{i}, z_{0}\right)=\sum_{i=1}^{d} \gamma_{i}(y) \lambda_{i}, \quad y \in \mathbb{R}^{d} .
\end{aligned}
$$

By (5.1), (5.3) and (5.10) we have $\gamma \equiv \rho$ for some $\rho \in \mathbb{R}^{d}$. Moreover, by (5.5), there exists a constant $\vartheta \in \mathbb{R}$ such that

$$
\theta\left(h_{0}+\sum_{i=1}^{d} y_{i} \lambda_{i}, z_{0}\right)=\vartheta, \quad y \in \mathbb{R}^{d} .
$$

Taking into account the HJM drift condition (5.2), inserting (5.10) into (5.9) yields

$$
\frac{d}{d x} h_{0}+\sum_{i=1}^{d} y_{i} \frac{d}{d x} \lambda_{i}+\frac{d}{d x} \Psi\left(\vartheta-\sum_{i=1}^{d} \rho_{i} \Lambda_{i}\right)=h_{0}^{\prime}+\sum_{i=1}^{d} \mu_{i}(y) \lambda_{i}, \quad y \in \mathbb{R}^{d} .
$$

Differentiating with respect to $y_{i}$ for $i=1, \ldots, d$ provides (5.8).

Conversely, suppose conditions (5.7), (5.8) are fulfilled. Then we have (2.15) and there exist $\left(a_{i j}\right)_{i, j=1, \ldots, d} \subset \mathbb{R}$ such that

$$
\frac{d}{d x} \lambda_{i}=\sum_{j=1}^{d} a_{i j} \lambda_{j}, \quad i=1, \ldots, d .
$$

Let $\left(h_{0}, z_{0}\right) \in \mathcal{D}(d / d x) \times \mathbb{R}^{m}$ and $t_{0} \in \mathbb{R}_{+}$be arbitrary. Since the vector field $\Theta$ defined in $(2.10)$ is of class $C_{\text {lip }}^{1}\left(\mathcal{H}_{\beta}\right)$, there exists, according to [31, Thm. 6.1.2, Thm. 6.1.5], a unique solution $\psi=\left(\psi_{1}, \psi_{2}\right) \in C^{1}\left(\mathbb{R}_{+} ; \mathcal{H}_{\beta}\right)$ with $\psi\left(\mathbb{R}_{+}\right) \subset \mathcal{D}(\mathcal{A})$ of the deterministic evolution equation

$$
\left\{\begin{array}{l}
\psi^{\prime}(t)=\mathcal{A} \psi(t)+\Theta(\psi(t)) \\
\psi(0)=h_{0}
\end{array}\right.
$$

where $\mathcal{A}$ is defined according to (2.10). In particular, relation (2.14) is fulfilled and we have

$$
\psi_{1}^{\prime}(t)=\frac{d}{d x} \psi_{1}(t)+\alpha(\psi(t)), \quad t \geq 0 .
$$

We define $\mu, \gamma \in C^{0,1,1}\left(\mathbb{R}_{+} \times \mathbb{R}^{d} \times \mathbb{R}^{m} ; \mathbb{R}^{d}\right)$ as

$$
\begin{aligned}
\mu_{i}(t, y, z) & :=\sum_{j=1}^{d} a_{j i} y_{j}, \quad i=1, \ldots, d \\
\gamma_{i}(t, y, z) & :=\Phi_{i}(\psi(t), z), \quad i=1, \ldots, d .
\end{aligned}
$$

Due to (5.1) and (5.3), condition (2.17) is satisfied. By (5.3), (5.4) and (5.5), (5.6), for all $h \in H_{\beta}, y \in \mathbb{R}^{d}$ and $z, w \in \mathbb{R}^{m}$ we have

$$
\begin{aligned}
& \alpha\left(h+\sum_{i=1}^{d} y_{i} \lambda_{i}, z+w\right)=-\sum_{i=1}^{d} \Phi_{i}\left(h+\sum_{j=1}^{d} y_{j} \lambda_{j}, z+w\right) \lambda_{i} \\
& \quad \times \Psi^{\prime}\left(\theta\left(h+\sum_{j=1}^{d} y_{j} \lambda_{j}, z+w\right)-\sum_{i=1}^{d} \Phi_{i}\left(h+\sum_{j=1}^{d} y_{j} \lambda_{j}, z+w\right) \Lambda_{i}\right) \\
& =-\sum_{i=1}^{d} \Phi_{i}(h, z) \lambda_{i} \Psi^{\prime}\left(\theta(h, z)-\sum_{i=1}^{d} \Phi_{i}(h, z) \Lambda_{i}\right)=\alpha(h, z) .
\end{aligned}
$$


Using (5.12), (5.15) and (5.11), for all $(t, y, z) \in \mathbb{R}_{+} \times \mathbb{R}^{d} \times \mathbb{R}^{m}$ we get

$$
\begin{aligned}
\nu\left(\psi_{1}(t)+\sum_{i=1}^{d} y_{i} \lambda_{i}, z\right) & =\frac{d}{d x}\left(\psi_{1}(t)+\sum_{i=1}^{d} y_{i} \lambda_{i}\right)+\alpha\left(\psi_{1}(t)+\sum_{i=1}^{d} y_{i} \lambda_{i}, z\right) \\
& =\psi_{1}^{\prime}(t)-\alpha(\psi(t))+\sum_{i=1}^{d} y_{i} \frac{d}{d x} \lambda_{i}+\alpha(\psi(t)) \\
& =\psi_{1}^{\prime}(t)+\sum_{i=1}^{d}\left(\sum_{j=1}^{d} a_{j i} y_{j}\right) \lambda_{i}=\psi_{1}^{\prime}(t)+\sum_{i=1}^{d} \mu_{i}(t, y, z) \lambda_{i},
\end{aligned}
$$

showing (2.16). By Corollary 2.8, the foliation $\left(M_{t}^{\left(h_{0}, z_{0}\right)} \times \mathbb{R}^{m}\right)_{t \geq 0}$ generated by $\left\langle\lambda_{1}, \ldots, \lambda_{d}\right\rangle$ with parametrization $\psi_{1}$ is invariant for the HJMM equation (1.4)

Now, we assume that the volatility $\sigma$ in the HJMM equation (1.4) is of the form

$$
\sigma(h, z)=\sum_{i=1}^{p} \Phi_{i}(h, z) \lambda_{i}, \quad(h, z) \in \mathcal{H}_{\beta}
$$

for some positive integer $p \in \mathbb{N}$, where $\Phi_{1}, \ldots, \Phi_{p}: \mathcal{H}_{\beta} \rightarrow \mathbb{R}$ and $\lambda_{1}, \ldots, \lambda_{p} \in H_{\beta^{\prime}}^{0}$ satisfy exactly the same conditions as stated below (5.1).

5.2. Proposition. Suppose $\sigma \not \equiv 0$, the HJMM equation (1.4) has an affine realization generated by some finite dimensional subspace $V \subset H_{\beta}$, for all $i=1, \ldots, p$ we have

$$
D \Phi_{i}(h, z)(v, w)=0, \quad(h, z) \in \mathcal{H}_{\beta} \text { and }(v, w) \in V \times \mathbb{R}^{m},
$$

for all $(h, z) \in \mathcal{H}_{\beta}$ with $\left(\Phi_{1}(h, z), \ldots, \Phi_{p}(h, z)\right) \neq 0$ we have

$$
D \theta(h, z)(v, w)=0, \quad(v, w) \in V \times \mathbb{R}^{m}
$$

and there exist $\left(h_{1}, z_{1}\right), \ldots,\left(h_{p}, z_{p}\right) \in \mathcal{H}_{\beta}$ such that $\sigma\left(h_{1}, z_{1}\right), \ldots, \sigma\left(h_{p}, z_{p}\right)$ are linearly independent. Then, $\lambda_{1}, \ldots, \lambda_{p}$ are quasi-exponential and we have

$$
\left\langle(d / d x)^{n} \lambda_{1}: n \in \mathbb{N}_{0}\right\rangle+\ldots+\left\langle(d / d x)^{n} \lambda_{p}: n \in \mathbb{N}_{0}\right\rangle \subset V .
$$

Proof. Lemma 2.10 yields that $\sigma(h, z) \in V$ for all $(h, z) \in \mathcal{H}_{\beta}$. Since the vectors $\sigma\left(h_{1}, z_{1}\right), \ldots, \sigma\left(h_{p}, z_{p}\right)$ are linearly independent, we obtain $\lambda_{1}, \ldots, \lambda_{p} \in V$, because relation (5.16) yields that

$$
\left\langle\lambda_{1}, \ldots, \lambda_{p}\right\rangle=\left\langle\sigma\left(h_{1}, z_{1}\right), \ldots, \sigma\left(h_{p}, z_{p}\right)\right\rangle .
$$

Set $d:=\operatorname{dim} V$ and choose $\lambda_{p+1}, \ldots, \lambda_{d} \in V$ such that $\left\{\lambda_{1}, \ldots, \lambda_{d}\right\}$ is a basis of $V$. Then, the volatility $\sigma$ is of the type (5.1) with $\Phi_{i} \equiv 0$ for $i=d+1, \ldots, p$. Since (5.3)(5.6) are satisfied due to (5.17), (5.18), Lemma 5.1 implies (5.7), (5.8). Relation (5.8) yields (5.19), and, together with Lemma 2.11 , we obtain that $\lambda_{1}, \ldots, \lambda_{p}$ are quasi-exponential.

5.3. Theorem. Suppose $\sigma \not \equiv 0$, the functions $\lambda_{1}, \ldots, \lambda_{p}$ are quasi-exponential, for all $i=1, \ldots, p$ and for $(h, z) \in \mathcal{H}_{\beta}$ we have

$$
\begin{aligned}
D \Phi_{i}(h, z)\left((d / d x)^{n} \lambda_{j}, 0\right) & =0, \quad j=1, \ldots, p \text { and } n \in \mathbb{N}_{0} \\
D \Phi_{i}(h, z)\left(0, e_{k}\right) & =0, \quad k=1, \ldots, m
\end{aligned}
$$

and for all $(h, z) \in \mathcal{H}_{\beta}$ with $\left(\Phi_{1}(h, z), \ldots, \Phi_{p}(h, z)\right) \neq 0$ we have

$$
\begin{aligned}
D \theta(h, z)\left((d / d x)^{n} \lambda_{j}, 0\right) & =0, \quad j=1, \ldots, p \text { and } n \in \mathbb{N}_{0} \\
D \theta(h, z)\left(0, e_{k}\right) & =0, \quad k=1, \ldots, m .
\end{aligned}
$$


Then, the HJMM equation (1.4) has an affine realization generated by the finite dimensional subspace

$$
V=\left\langle(d / d x)^{n} \lambda_{1}: n \in \mathbb{N}_{0}\right\rangle+\ldots+\left\langle(d / d x)^{n} \lambda_{p}: n \in \mathbb{N}_{0}\right\rangle .
$$

Proof. Since $\lambda_{1}, \ldots, \lambda_{p}$ are quasi-exponential, the linear space $V$ is finite dimensional, and we have

$$
V \subset \mathcal{D}(d / d x) \text { and } \quad(d / d x) v \in V \text { for all } v \in V .
$$

Set $d:=\operatorname{dim} V$ and choose $\lambda_{p+1}, \ldots, \lambda_{d} \in V$ such that $\left\{\lambda_{1}, \ldots, \lambda_{d}\right\}$ is a basis of $V$. Then, the volatility $\sigma$ is of the type (5.1) with $\Phi_{i} \equiv 0$ for $i=d+1, \ldots, p$. Conditions (5.3)-(5.6) are satisfied by (5.20)-(5.23), and relations (5.7), (5.8) are fulfilled due to (5.25). According to Lemma 5.1, the HJMM equation (1.4) has an affine realization generated by $V$.

Theorem 5.3 is a first central result concerning the existence of affine realizations for Lévy term structure models, as it provides sufficient conditions in terms of the volatility $\sigma$. The functions $\lambda_{1}, \ldots, \lambda_{p}$ are assumed to be quasi-exponential, which, by Proposition 5.2, is essentially also necessary under conditions (5.20)-(5.23).

At this point, conditions (5.20)-(5.23) look rather ad-hoc, but, as we will see in Theorems 6.1 and 6.3 below, we cannot expect more general volatility structures for Lévy term structure models with an affine realization.

We close this section with an example. Suppose the volatility $\sigma$ and the market price of risk $\theta$ in the HJMM equation (1.4) are constant, i.e., we have $\sigma \equiv \lambda$ with $\lambda \in H_{\beta^{\prime}}^{0}, \lambda \neq 0$ and $\theta \equiv \vartheta$ with $\vartheta \in \mathbb{R}$ such that $\vartheta-\Lambda \in \mathcal{O}$. Then, Assumption 3.2 is fulfilled.

5.4. Corollary. The following statements are equivalent:

(1) The HJMM equation (1.4) has an affine realization.

(2) The HJMM equation (1.4) with $\theta \equiv 0$ has an affine realization.

(3) $\lambda$ is quasi-exponential.

Proof. This is an immediate consequence of Proposition 5.2 and Theorem 5.3.

Hence, for constant volatilities the statement of Proposition 4.1 also holds true for driving Lévy processes with jumps. As we shall see in the upcoming section, it fails for more complex volatility structures.

\section{Constant direction volatility}

In this section, we study the existence of affine realizations for Lévy term structure models with constant direction volatility, that is, we assume that the volatility $\sigma$ in the HJMM equation (1.4) is of the form

$$
\sigma(h, z)=\Phi(h, z) \lambda, \quad(h, z) \in \mathcal{H}_{\beta},
$$

where $\Phi: \mathcal{H}_{\beta} \rightarrow \mathbb{R}$ is a functional and $\lambda \in H_{\beta^{\prime}}^{0}$ with $\lambda \neq 0$. We assume that $\Phi \in C_{b}^{1}\left(\mathcal{H}_{\beta} ; \mathbb{R}\right)$ and $(\theta-\mathcal{I} \sigma)\left(\mathcal{H}_{\beta}\right) \subset \mathcal{O}$. Then, Assumption 3.2 is fulfilled. Recall that $F$ denotes the Lévy measure of the driving Lévy process $X$ in (1.4).

Let us first consider the situation where the market price of risk vanishes.

6.1. Theorem. Suppose $\theta \equiv 0, F(\mathbb{R}) \neq 0$ and $\Phi \not \equiv 0$. Then, the HJMM equation (1.4) has an affine realization if and only if $\lambda$ is quasi-exponential and for all $(h, z) \in \mathcal{H}_{\beta}$ we have

$$
\begin{aligned}
D \Phi(h, z)\left((d / d x)^{n} \lambda, 0\right) & =0, & & n \in \mathbb{N}_{0} \\
D \Phi(h, z)\left(0, e_{k}\right) & =0, & & k=1, \ldots, m
\end{aligned}
$$


Proof. This follows from a straightforward extension of the arguments in the proof of [38, Thm. 4.1].

Theorem 6.1 shows that for the existence of an affine realization we get the additional restrictions (6.2), (6.3) on $\Phi$, which means that for each forward curve $h \in H_{\beta}$ the functional $\Phi$ is constant in the direction of the finite dimensional linear space $V$, which generates the affine realization. We shall prove in Theorem 6.3 below that also an analogous condition on the market price of risk $\theta$ must be satisfied. For this purpose, we prepare an auxiliary result.

6.2. Lemma. Let $a, b \in \mathbb{R}$ with $a<b$ and let $p \in \mathbb{N}$ be arbitrary. Let $f_{1}, \ldots, f_{p}$ : $(a, b) \rightarrow \mathbb{C}$ be linearly independent, real-analytic functions. Then, for all $c, d \in \mathbb{R}$ with $c<d$ and $(c, d) \subset(a, b)$ there exist $z_{1}, \ldots, z_{p} \in(c, d)$ such that

$$
\operatorname{det}\left(\begin{array}{ccc}
f_{1}\left(z_{1}\right) & \cdots & f_{1}\left(z_{p}\right) \\
\vdots & & \vdots \\
f_{p}\left(z_{1}\right) & \cdots & f_{p}\left(z_{p}\right)
\end{array}\right) \neq 0 .
$$

Proof. Let $c, d \in \mathbb{R}$ with $c<d$ be arbitrary. We first suppose $p=1$. Since $f_{1} \not \equiv 0$ on $(a, b)$ by the assumed linear independence, according to the identity theorem for analytic functions there exists $z_{1} \in(c, d)$ with $f_{1}\left(z_{1}\right) \neq 0$. For $p \geq 2$ we proceed by induction and suppose there exist $z_{1}, \ldots, z_{p-1} \in(c, d)$ such that

$$
\operatorname{det}\left(\begin{array}{ccc}
f_{1}\left(z_{1}\right) & \cdots & f_{1}\left(z_{p-1}\right) \\
\vdots & & \vdots \\
f_{p-1}\left(z_{1}\right) & \cdots & f_{p-1}\left(z_{p-1}\right)
\end{array}\right) \neq 0 .
$$

Then, the function $g:(a, b) \rightarrow \mathbb{C}$ given by

$$
g(z)=\operatorname{det}\left(\begin{array}{cccc}
f_{1}\left(z_{1}\right) & \cdots & f_{1}\left(z_{p-1}\right) & f_{1}(z) \\
\vdots & & \vdots & \vdots \\
f_{p-1}\left(z_{1}\right) & \cdots & f_{p-1}\left(z_{p-1}\right) & f_{p-1}(z) \\
f_{p}\left(z_{1}\right) & \cdots & f_{p}\left(z_{p-1}\right) & f_{p}(z)
\end{array}\right)
$$

is also real-analytic, and it is of the form

$$
g(z)=\sum_{i=1}^{p} \xi_{i} f_{i}(z), \quad z \in(a, b)
$$

with $\xi_{1}, \ldots, \xi_{p} \in \mathbb{R}$ and $\xi_{p} \neq 0$. Since $f_{1}, \ldots, f_{p}$ are linearly independent, we have $g \not \equiv 0$ on $(a, b)$. Since $g$ is real-analytic, by the identity theorem for analytic functions there exists $z_{p} \in(c, d)$ with $g\left(z_{p}\right) \neq 0$, which finishes the proof.

In analogy to $(2.19)$ we say that the cumulant generating function $\Psi$ is quasiexponential if

$$
\operatorname{dim}\left\langle\Psi^{(n)}: n \in \mathbb{N}_{0}\right\rangle<\infty .
$$

6.3. Theorem. Suppose that $\Phi \not \equiv 0, \Psi$ is not quasi-exponential, the HJMM equation (1.4) has an affine realization generated by some finite dimensional subspace $V \subset$ $H_{\beta}$, and

$$
D \Phi(h, z)(v, w)=0, \quad(h, z) \in \mathcal{H}_{\beta} \text { and }(v, w) \in V \times \mathbb{R}^{m} .
$$

Then, for all $(h, z) \in \mathcal{H}_{\beta}$ with $\Phi(h, z) \neq 0$ we have

$$
D \theta(h, z)(v, w)=0, \quad(v, w) \in V \times \mathbb{R}^{m} .
$$


Proof. Set $d:=\operatorname{dim} V$. Since $\Phi \not \equiv 0$ and $\mathcal{D}(d / d x)$ is dense in $H_{\beta}$, condition (2.13) from Corollary 2.7 and (6.1) yield that $\lambda \in V$. Set $\lambda_{1}:=\lambda$ and choose $\lambda_{2}, \ldots, \lambda_{d} \in$ $V$ such that $\left\{\lambda_{1}, \ldots, \lambda_{d}\right\}$ is a basis of $V$. Now, let $\left(h_{0}, z_{0}\right) \in \mathcal{D}(d / d x) \times \mathbb{R}^{m}$ with $\Phi\left(h_{0}, z_{0}\right) \neq 0$ be arbitrary. We apply Corollary 2.8 to the invariant foliation $\left(M_{t}^{\left(h_{0}, z_{0}\right)} \times \mathbb{R}^{m}\right)_{t \geq 0}$, implying $\lambda_{1}, \ldots, \lambda_{d} \in \mathcal{D}(d / d x)$ and the existence of $h_{0}^{\prime} \in H_{\beta}$ and $\mu \in C^{1}\left(\mathbb{R}^{d} \times \mathbb{R}^{m} ; \mathbb{R}^{d}\right), \gamma \in C^{1}\left(\mathbb{R}^{d} \times \mathbb{R}^{m} ; \mathbb{R}\right)$ such that

$$
\begin{aligned}
& \nu\left(h_{0}+\sum_{i=1}^{d} y_{i} \lambda_{i}, z\right)=h_{0}^{\prime}+\sum_{i=1}^{d} \mu_{i}(y, z) \lambda_{i}, \quad(y, z) \in \mathbb{R}^{d} \times \mathbb{R}^{m} \\
& \sigma\left(h_{0}+\sum_{i=1}^{d} y_{i} \lambda_{i}, z\right)=\gamma(y, z) \lambda, \quad(y, z) \in \mathbb{R}^{d} \times \mathbb{R}^{m}
\end{aligned}
$$

where, in view of (6.1), the mapping $\gamma: \mathbb{R}^{d} \times \mathbb{R}^{m} \rightarrow \mathbb{R}$ is given by

$$
\gamma(y, z)=\Phi\left(h_{0}+\sum_{i=1}^{d} y_{i} \lambda_{i}, z\right), \quad(y, z) \in \mathbb{R}^{d} \times \mathbb{R}^{m} .
$$

In view of (6.4), there exists a constant $\rho \in \mathbb{R}$ such that $\gamma \equiv \rho$, and, since $\Phi\left(h_{0}, z_{0}\right) \neq$ 0 , we have $\rho \neq 0$. We define $\vartheta: \mathbb{R}^{d} \times \mathbb{R}^{m} \rightarrow \mathbb{R}$ as

$$
\vartheta(y, z):=\theta\left(h_{0}+\sum_{i=1}^{d} y_{i} \lambda_{i}, z\right), \quad(y, z) \in \mathbb{R}^{d} \times \mathbb{R}^{m} .
$$

Taking into account the drift condition (1.5), inserting (6.7) into (6.6) gives us

$$
\frac{d}{d x} h_{0}+\sum_{i=1}^{d} y_{i} \frac{d}{d x} \lambda_{i}+\frac{d}{d x} \Psi(\vartheta(y, z)-\rho \Lambda)=h_{0}^{\prime}+\sum_{i=1}^{d} \mu_{i}(y, z) \lambda_{i}, \quad(y, z) \in \mathbb{R}^{d} \times \mathbb{R}^{m} .
$$

Differentiating with respect to $y_{k}$ and $z_{l}$ we obtain

$$
\frac{d}{d x} \lambda_{k}+\frac{d}{d x}\left(\frac{\partial}{\partial y_{k}} \vartheta(y, z) \Psi^{\prime}(\vartheta(y, z)-\rho \Lambda)\right)=\sum_{i=1}^{d} \frac{\partial}{\partial y_{k}} \mu_{i}(y, z) \lambda_{i}, \quad k=1, \ldots, d
$$

and

$$
\frac{d}{d x}\left(\frac{\partial}{\partial z_{l}} \vartheta(y, z) \Psi^{\prime}(\vartheta(y, z)-\rho \Lambda)\right)=\sum_{i=1}^{d} \frac{\partial}{\partial z_{l}} \mu_{i}(y, z) \lambda_{i}, \quad l=1, \ldots, m
$$

for all $(y, z) \in \mathbb{R}^{d} \times \mathbb{R}^{m}$. Applying the integral operator $h \mapsto \int_{0}^{\bullet} h(\eta) d \eta$ yields

$$
\begin{aligned}
& \lambda_{k}-\lambda_{k}(0)+\frac{\partial}{\partial y_{k}} \vartheta(y, z) \Psi^{\prime}(\vartheta(y, z)-\rho \Lambda)-\frac{\partial}{\partial y_{k}} \vartheta(y, z) \Psi^{\prime}(\vartheta(y, z)) \\
& -\sum_{i=1}^{d} \frac{\partial}{\partial y_{k}} \mu_{i}(y, z) \Lambda_{i}=0, \quad k=1, \ldots, d
\end{aligned}
$$

and

$$
\begin{aligned}
& \frac{\partial}{\partial z_{k}} \vartheta(y, z) \Psi^{\prime}(\vartheta(y, z)-\rho \Lambda)-\frac{\partial}{\partial z_{k}} \vartheta(y, z) \Psi^{\prime}(\vartheta(y, z)) \\
& -\sum_{i=1}^{d} \frac{\partial}{\partial z_{l}} \mu_{i}(y, z) \Lambda_{i}=0, \quad l=1, \ldots, m
\end{aligned}
$$

for all $(y, z) \in \mathbb{R}^{d} \times \mathbb{R}^{m}$. We claim that $\vartheta$ is constant. Suppose, on the contrary, that $\vartheta$ is not constant. Then, there exist $k \in\{1, \ldots, d+m\}$ and $\left(y_{0}, z_{0}\right) \in \mathbb{R}^{d} \times \mathbb{R}^{m}$ such that $\partial_{k} \vartheta\left(y_{0}, z_{0}\right) \neq 0$. Hence, there exists $\delta>0$ such that

$$
\partial_{k} \vartheta\left(\left(y_{0}, z_{0}\right)+s e_{k}\right) \neq 0 \text { for all } s \in(-\delta, \delta) \text {. }
$$


Set $p:=2 d+2$. The functions $\Psi^{\prime}, \ldots, \Psi^{(p+1)}$ are analytic (see Section 3 ) and, since $\Psi$ is not quasi-exponential by assumption, they are linearly independent, as otherwise the condition

$$
\operatorname{dim}\left\langle\Psi^{(n)}: n \in \mathbb{N}_{0}\right\rangle=\infty
$$

is not satisfied. By Lemma 6.2 there exist elements $s_{1}, \ldots, s_{p} \in(-\delta, \delta)$ such that $\operatorname{det} B \neq 0$, where $B \in \mathbb{R}^{p \times p}$ denotes the matrix

$$
B=\left(\begin{array}{ccc}
\Psi^{\prime}\left(w_{1}\right) & \cdots & \Psi^{\prime}\left(w_{p}\right) \\
\vdots & & \vdots \\
\Psi^{(p+1)}\left(w_{1}\right) & \cdots & \Psi^{(p+1)}\left(w_{p}\right)
\end{array}\right)
$$

with $w_{i}:=\vartheta\left(\left(y_{0}, z_{0}\right)+s_{i} e_{k}\right)$ for $i=1, \ldots, p$. We claim that the family

$$
\left\{\Psi^{\prime}\left(w_{i}-\rho \Lambda\right): i=1, \ldots, p\right\}
$$

is linearly independent in $C\left(\mathbb{R}_{+}\right)$. Indeed, let $\xi_{1}, \ldots, \xi_{m} \in \mathbb{R}$ be such that

$$
\sum_{i=1}^{p} \xi_{i} \Psi^{\prime}\left(w_{i}-\rho \Lambda\right)=0 \quad \text { in } C\left(\mathbb{R}_{+}\right)
$$

Then we have

$$
\sum_{i=1}^{p} \xi_{i} \Psi^{\prime}\left(w_{i}+x\right)=0, \quad x \in-\rho \Lambda\left(\mathbb{R}_{+}\right) .
$$

By the power series representation (3.3) there exists $\eta>0$ such that $-\rho \Lambda\left(\mathbb{R}_{+}\right) \subset$ $(-\eta, \eta)$ and

$$
\begin{aligned}
\sum_{i=1}^{p} \xi_{i} \Psi^{\prime}\left(w_{i}+x\right) & =\sum_{i=1}^{p} \xi_{i} \sum_{n=0}^{\infty} \frac{\Psi^{(n+1)}\left(w_{i}\right)}{n !} x^{n} \\
& =\sum_{n=0}^{\infty}\left(\sum_{i=1}^{p} \xi_{i} \frac{\Psi^{(n+1)}\left(w_{i}\right)}{n !}\right) x^{n}, \quad x \in(-\eta, \eta)
\end{aligned}
$$

and hence, we obtain

$$
\sum_{n=0}^{\infty}\left(\sum_{i=1}^{p} \xi_{i} \frac{\Psi^{(n+1)}\left(w_{i}\right)}{n !}\right) x^{n}=0, \quad x \in-\rho \Lambda\left(\mathbb{R}_{+}\right) .
$$

Since $\rho \neq 0, \lambda \neq 0$ and $\Lambda(0)=0$, there exists a sequence $\left(x_{n}\right)_{n \in \mathbb{N}} \subset-\rho \Lambda\left(\mathbb{R}_{+}\right)$with $x_{n} \neq 0, n \in \mathbb{N}$ and $x_{n} \rightarrow 0$. Therefore, the identity theorem for power series applies and yields

$$
\frac{1}{n !} \sum_{i=1}^{p} \xi_{i} \Psi^{(n+1)}\left(w_{i}\right)=0, \quad n \in \mathbb{N}_{0} .
$$

It follows that $B \xi=0$, where $\xi \in \mathbb{R}^{p}$ is the vector $\xi=\left(\xi_{1}, \ldots, \xi_{p}\right)^{\top}$. Since $\operatorname{det} B \neq 0$, we deduce that $\xi_{1}=\ldots=\xi_{p}=0$. Consequently, the family (6.13) is linearly independent in $C\left(\mathbb{R}_{+}\right)$. Thus, recalling that $p=2 d+2$, there exists $i \in\{1, \ldots, p\}$ such that

$$
\Psi^{\prime}\left(\vartheta\left(\left(y_{0}, z_{0}\right)+s_{i} e_{k}\right)-\rho \Lambda\right) \notin\left\langle 1, \lambda_{1}, \ldots, \lambda_{d}, \Lambda_{1}, \ldots, \Lambda_{d}\right\rangle .
$$

Now, equation (6.10), resp. equation (6.11), yields $\partial_{k} \vartheta\left(\left(y_{0}, z_{0}\right)+s_{i} e_{k}\right)=0$, which contradicts $(6.12)$. Consequently, $\vartheta$ is constant. Since $\left(h_{0}, z_{0}\right) \in \mathcal{D}(d / d x) \times \mathbb{R}^{m}$ with $\Phi\left(h_{0}, z_{0}\right) \neq 0$ was arbitrary, the definition (6.9) shows that (6.5) is valid for all $(h, z) \in \mathcal{D}(d / d x) \times \mathbb{R}^{m}$ with $\Phi(h, z) \neq 0$. Since $\mathcal{D}(d / d x)$ is dense in $H_{\beta}$ and $\theta \in C^{1}\left(\mathcal{H}_{\beta} ; \mathbb{R}\right)$, we deduce $(6.5)$ for all $(h, z) \in \mathcal{H}_{\beta}$. 
6.4. Remark. If the hypotheses of Theorem 6.3 are satisfied, then Proposition 5.2 further yields that $\lambda$ is quasi-exponential and

$$
\left\langle(d / d x)^{n} \lambda: n \in \mathbb{N}_{0}\right\rangle \subset V .
$$

Conversely, if $\lambda$ is quasi-exponential, for all $(h, z) \in \mathcal{H}_{\beta}$ we have (6.2), (6.3), and for all $(h, z) \in \mathcal{H}_{\beta}$ with $\Phi(h, z) \neq 0$ we have

$$
\begin{array}{rlrl}
D \theta(h, z)\left((d / d x)^{n} \lambda, 0\right) & =0, & & n \in \mathbb{N}_{0} \\
D \theta(h, z)\left(0, e_{k}\right) & =0, & k=1, \ldots, m,
\end{array}
$$

then, according to Theorem 5.3, the HJMM equation (1.4) has an affine realization generated by the finite dimensional subspace

$$
V=\left\langle(d / d x)^{n} \lambda: n \in \mathbb{N}_{0}\right\rangle .
$$

6.5. Remark. The assumption that $\Psi$ is not quasi-exponential is essential for Theorem 6.3. For example, let $X$ be a standard Poisson process, which has the cumulant generating function $\Psi(x)=e^{x}-1$. For $\sigma \equiv 1$ and an arbitrary function $\theta: \mathcal{H}_{\beta} \rightarrow \mathbb{R}$ for the market price of risk, the drift term (1.5) is given by

$$
\alpha(h, z)(x)=-e^{\theta(h, z)-x}=-e^{\theta(h, z)} e^{-x} .
$$

The corresponding HJMM equation (1.4) has a two-dimensional affine realization generated by the linear space $V=\left\langle 1, e^{-x}\right\rangle$. Indeed, for arbitrary $\left(h_{0}, z_{0}\right) \in$ $\mathcal{D}(d / d x) \times \mathbb{R}^{m}$ the foliation $M_{t}^{\left(h_{0}, z_{0}\right)}=S_{t} h_{0}+V, t \geq 0$ is invariant for (1.4). Conditions (2.11), (2.13) are satisfied, and condition (2.12) holds true, because $(d / d x) S_{t} h_{0}=(d / d t) S_{t} h_{0}$ and $(d / d x) v \in V$ for all $v \in V$. Consequently, Corollary 2.7 applies.

In summary, we have just seen that for the existence of an affine realization we obtain - compared to the Wiener process driven case - further restrictions on the function $\Phi$ and on the market price of risk $\theta$, which is due to the particular structure of the drift term $\alpha$ in (1.5).

\section{CONClusion}

We have investigated the existence of affine realizations for interest rate models driven by Lévy processes under real-world forward rate dynamics.

If the driving process $X$ is a Wiener process, then the term structure model admits an affine realization if and only if the corresponding model with $\theta \equiv 0$ (i.e. the market price of risk vanishes) admits an affine realization. Hence, for Wiener process driven models all known results concerning the existence of affine realizations for HJM interest rate models under an assumed risk-neutral probability measure transfer to interest rate models with real-world forward rate dynamics, even without assuming the existence of an equivalent risk-neutral probability measure.

If the driving Lévy process $X$ has jumps, then essentially all volatility structures with an affine realization represent a weighted sum of particular functionals with the weights being quasi-exponential. This is due to the particular structure of the drift term in the forward rate equation.

\section{REFERENCES}

[1] Baudoin, F., Teichmann, J. (2005): Hypoellipticity in infinite dimensions and an application in interest rate theory. Annals of Applied Probability 15(3), 1765-1777.

[2] Bhar, R., Chiarella, C. (1997): Transformation of Heath-Jarrow-Morton models to Markovian systems. The European Journal of Finance 3, 1-26.

[3] Björk, T. (2003): On the geometry of interest rate models. Paris-Princeton Lectures on Mathematical Finance, 133-215. 
[4] Björk, T., Christensen, C. (1999): Interest rate dynamics and consistent forward rate curves. Mathematical Finance 9(4), 323-348.

[5] Björk, T., Gombani, A. (1999): Minimal realizations of interest rate models. Finance and Stochastics 3(4), 413-432.

[6] Björk, T., Landén, C. (2002): On the construction of finite dimensional realizations for nonlinear forward rate models. Finance and Stochastics 6(3), 303-331.

[7] Björk, T., Landén, C., Svensson, L. (2004): Finite-dimensional Markovian realizations for stochastic volatility forward-rate models. Stochastic analysis with applications to mathematical finance. Proceedings of The Royal Society of London. Series A. Mathematical, Physical and Engineering Sciences 460(2041), 53-83.

[8] Björk, T., Svensson, L. (2001): On the existence of finite dimensional realizations for nonlinear forward rate models. Mathematical Finance 11(2), 205-243.

[9] Bruti-Liberati, N., Nikitopoulos-Sklibosiois, C., Platen, E. (2010): Real-world jump-diffusion term structure models. Quantitative Finance 10(1), 23-37.

[10] Chiarella, C., Kwon, O. K. (2001): Forward rate dependent Markovian transformations of the Heath-Jarrow-Morton term structure model. Finance and Stochastics 5(2), 237-257.

[11] Chiarella, C., Kwon, O. K. (2003): Finite dimensional affine realizations of HJM models in terms of forward rates and yields. Review of Derivatives Research 6(3), 129-155.

[12] Duffie, D., Kan, R. (1996): A yield-factor model of interest rates. Mathematical Finance 6(4), 379-406.

[13] Eberlein, E., Jacod, J., Raible, S. (2005): Lévy term structure models: no-arbitrage and completeness. Finance and Stochastics 9(1), 67-88.

[14] Eberlein, E., Kluge, W. (2006): Exact pricing formulae for caps and swaptions in a Lévy term structure model. Journal of Computational Finance 9(2), 99-125.

[15] Eberlein, E., Kluge, W. (2006): Valuation of floating range notes in Lévy term structure models. Mathematical Finance 16(2), 237-254.

[16] Eberlein, E., Kluge, W. (2007): Calibration of Lévy term structure models. In Advances in Mathematical Finance: In Honor of Dilip Madan, M. Fu, R. A. Jarrow, J.-Y. Yen, and R. J. Elliott (Eds.), Birkhäuser, pp. 155-180.

[17] Eberlein, E., Özkan, F. (2003): The defaultable Lévy term structure: ratings and restructuring. Mathematical Finance 13(2), 277-300.

[18] Eberlein, E., Raible, S. (1999): Term structure models driven by general Lévy processes. Mathematical Finance 9(1), 31-53.

[19] Filipović, D. (2001): Consistency Problems for Heath-Jarrow-Morton Interest Rate Models. Berlin: Springer.

[20] Filipović, D., Tappe, S. (2008): Existence of Lévy term structure models. Finance and Stochastics 12(1), 83-115.

[21] Filipović, D., Teichmann, J. (2003): Existence of invariant manifolds for stochastic equations in infinite dimension. Journal of Functional Analysis 197(2), 398-432.

[22] Filipović, D., Teichmann, J. (2004): On the geometry of the term structure of interest rates. Proceedings of The Royal Society of London. Series A. Mathematical, Physical and Engineering Sciences 460(2041), 129-167.

[23] Gapeev, P., Küchler, U. (2006) On Markovian short rates in term structure models driven by jump-diffusion processes. Statistics and Decisions 24(2), 255-271.

[24] Heath, D., Jarrow, R., Morton, A. (1992): Bond pricing and the term structure of interest rates: a new methodology for contingent claims valuation. Econometrica 60(1), 77-105.

[25] Hyll, M. (2000): Affine term structures and short-rate realizations of forward rate models driven by jump-diffusion processes. In Essays on the term structure of interest rates $\mathrm{PhD}$ thesis, Stockholm School of Economics.

[26] Inui, K., Kijima, M. (1998): A Markovian framework in multi-factor Heath-Jarrow-Morton models. Journal of Financial and Quantitative Analysis 33(3), 423-440.

[27] Jeffrey, A. (1995): Single factor Heath-Jarrow-Morton term structure models based on Markov spot interest rate dynamics. Journal of Financial and Quantitative Analysis 30(4), 619-642.

[28] Küchler, U., Naumann, E. (2003) Markovian short rates in a forward rate model with a general class of Lévy processes. Discussion Paper 6, Sonderforschungsbereich 373, Humboldt University Berlin.

[29] Marinelli, C. (2010): Local well-posedness of Musiela's SPDE with Lévy noise. Mathematical Finance 20(3), 341-363.

[30] Musiela, M. (1993): Stochastic PDEs and term structure models. Journées Internationales de Finance, IGR-AFFI, La Baule.

[31] Pazy, A. (1983): Semigroups of linear operators and applications to partial differential equations. Springer, New York. 
[32] Peszat, S., Zabczyk, J. (2007): Heath-Jarrow-Morton-Musiela equation of bond market. Preprint IMPAN 677, Warsaw. (www.impan.gov.pl/EN/Preprints/index.html)

[33] Platen, E., Christensen, M. M. (2005): A general benchmark model for stochastic jump sizes. Stochastic Analysis and Applications 23(5), 1017-1044.

[34] Platen, E., Heath, D. (2006): A Benchmark Approach to Quantitative Finance. Springer, Berlin.

[35] Ritchken, P., Sankarasubramanian, L. (1995): Volatility structures of forward rates and the dynamics of the term structure. Mathematical Finance 5(1), 55-72.

[36] Rudin, W. (1991): Functional Analysis. McGraw-Hill, Second Edition.

[37] Tappe, S. (2010): An alternative approach on the existence of affine realizations for HJM term structure models. Proceedings of The Royal Society of London. Series A. Mathematical, Physical and Engineering Sciences 466 (2122), 3033-3060.

[38] Tappe, S. (2011): Existence of affine realizations for Lévy term structure models. Preprint, submitted. (www.math.ethz.ch/ tappes/FDR_Levy.pdf)

School of Mathematical Sciences and School of Finance and Economics, University of Technology Sydney, PO Box 123, Broadway, NSW 2007, Australia

E-mail address: eckhard.platen@uts.edu.au

ETH Zürich, Department of Mathematics, RÄmistrasse 101, CH-8092 Zürich, SwitzerLAND

E-mail address: stefan.tappe@math.ethz.ch 